\title{
Resección de leiomioma intravascular e intracardiaco: reporte de un caso
}

\author{
Resection of intravascular and intracardiac leiomyoma: a case report
}

Ressecção de leiomioma intravascular e intracardíaco: um relato de caso

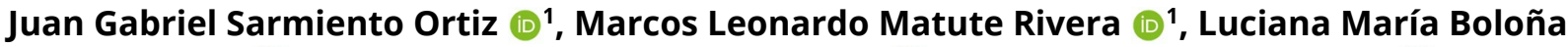 Gilbert (1) 1, Lorena Jaqueline Aragón Quijano (1) 2, Diana María Núñez Parra (1) ${ }^{1}$}

'Servicio de Cirugía General, Clínica Guayaquil, Guayaquil-Ecuador;

2 Servicio de Medicina Interna, Clínica Guayaquil, Guayaquil Ecuador.

\section{Correspondencia a:}

Juan Gabriel Sarmiento Ortiz, gabosarmiento1@gmail.com

Recibido: 1 de julio, 2021

Aceptado: 9 de septiembre, 2021

Publicado: 18 de noviembre, 2021

\section{CASO CLÍNICO}

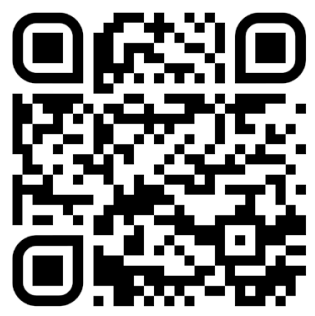

Escanea en tu dispositivo móvil o revisa este artículo en: https:// revistaclinicaguayaquil.org

\section{RESUMEN}

La leiomiomatosis extrauterina es una entidad rara que afecta principalmente a mujeres premenopáusicas. Se presenta el caso de una mujer de 46 años de edad, con diagnóstico de tumor intravascular e intracardiaco, así como hidronefrosis derecha, el mismo que fue tratado mediante resección quirúrgica.

Palabras clave: Leiomioma; Leiomiomatosis; Vena cava; Trombosis venosa

\begin{abstract}
Extrauterine leiomyomatosis is a rare entity that mainly affects premenopausal women. We present the case of a 46-year-old woman with a diagnosis of an intravascular and intracardiac tumor, as well as right hydronephrosis, the same who was treated by surgical resection.
\end{abstract}

Key words: Leiomyoma; Leiomyomatosis; Vena cava; Venous thrombosis

\section{RESUMO}

A leiomiomatose extrauterina é uma entidade rara que afeta principalmente as mulheres na pré-menopausa. Apresentamos o caso de uma mulher de 46 anos com diagnóstico de tumor intravascular e intracardíaco e hidronefrose direita, que foi tratada por ressecção cirúrgica.

Palavras-chave: Leiomioma; Leiomiomatose; Veia cava; Trombose venosa

\section{INTRODUCCIÓN}

La leiomiomatosis intravenosa (LIV) con extensión intracardíaca fue descrita por primera vez por Hirschfield en 1896 (1); corresponde a un leiomioma uterino típico con localizaciones inusuales, que se extiende hacia el sistema venoso a través de los canales uterinos y que puede progresar a las cámaras cardíacas $(2,3)$. Predominantemente afecta a mujeres premenopáusicas, caucásicas, con antecedentes de leiomiomas uterinos o histerectomía (1).

\section{DESCRIPCIÓN DEL CASO CLÍNICO}

Paciente femenina de 46 años, con antecedentes de miomatosis uterina, resuelta con histerectomía total 2 años previos a cuadro actual, además de hipertensión arterial y uso de anticoagulantes orales, sin un motivo referido por la paciente. Acude con cuadro 
clínico caracterizado por disnea de medianos esfuerzos como única sintomatología; es derivada al hospital con diagnóstico presuntivo de mixoma en aurícula derecha. En el examen físico los parámetros fueron normales. En el ecocardiograma se evidenció aumento severo de aurícula derecha (volumen estimado en $50 \mathrm{ml} / \mathrm{m} 2$ ), presencia de masa localizada en aurícula derecha de contextura heterogénea que aparentemente proviene de la vena cava inferior, mide aproximadamente 80 x 35 mm, y protruye en fase sistólica dentro del ventrículo derecho; el estudio Doppler y mapeo con color mostró regurgitación leve de la válvula tricúspide (Figura 1A). La angiotomografía de tórax y abdomen reportó hidronefrosis derecha, además de signos de trombosis de la vena renal que se extiende desde la vena renal derecha, por la vena cava hacia la aurícula derecha (Figura 1 B y C).

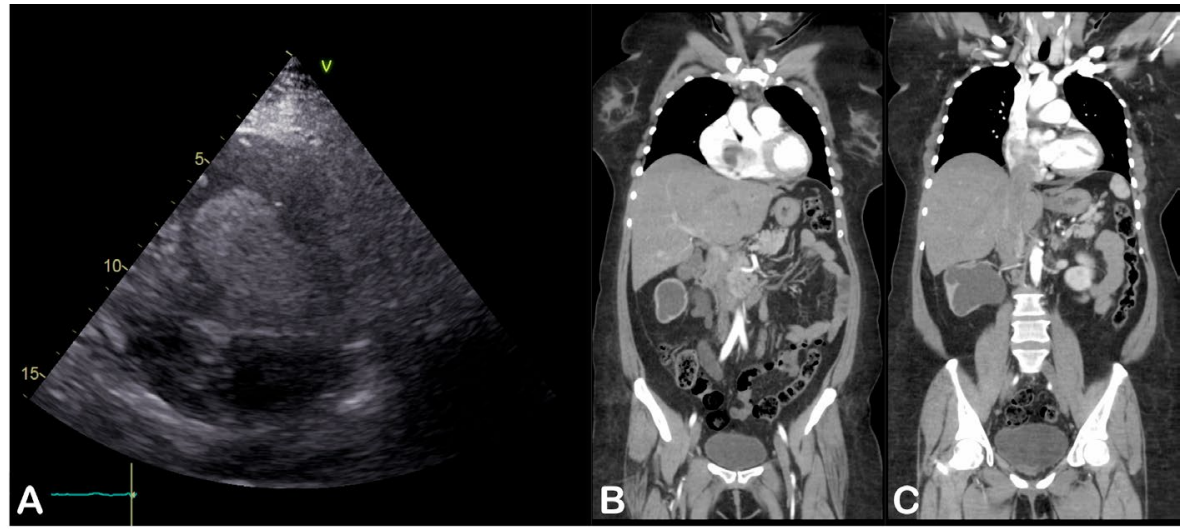

Figura 1. A. Ecocardiograma, se evidenció aumento severo de aurícula derecha, presencia de masa en aurícula derecha de contextura heterogénea que protruye en fase sistólica dentro del ventrículo derecho. B y C. Angiotomografía de tórax y abdomen, corte coronal. Signos de trombosis desde la vena renal derecha hacia la vena cava y la aurícula derecha; se evidencia hidronefrosis derecha.

Se decide su resolución quirúrgica; en un mismo tiempo quirúrgico se realizó un abordaje toracoabdominal, se utilizó bomba de circulación extracorpórea, además de parada cardiaca con hipotermia leve. Se evidenció a nivel cardiaco un tumor de contextura cauchosa, que ocupa la totalidad de la aurícula derecha y se prolonga a la vena cava inferior y se extendía hasta las venas renales (Figura 2); además, en abdomen se observó lesión del uréter derecho asociado a hidronefrosis derecha grado 4. Se realiza exéresis de dicha masa en forma completa, así como nefrectomía derecha. El estudio histopatológico reportó leiomiomatosis intravascular. Se realizaron estudios de inmunohistoquímica: desmina (+), SMA (+), ER (+), y Ki-67 (+) $<2 \%$, corroborando el diagnóstico de leiomiomatosis intravascular.

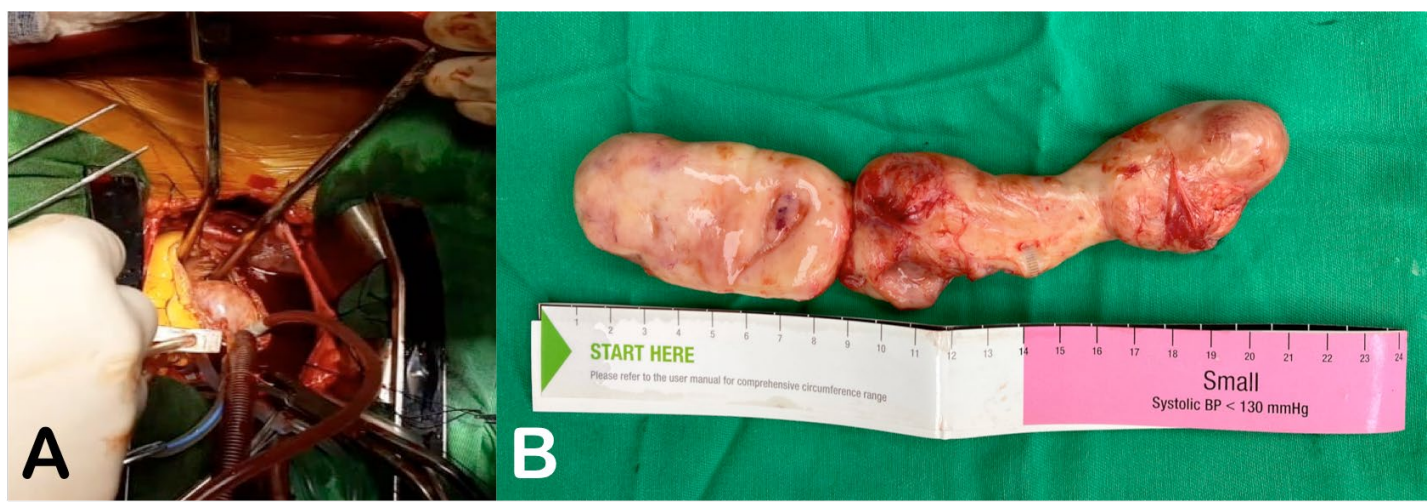

Figura 2. A. Exposición intraoperatoria de aurícula derecha, donde se evidencia presencia de tumor. B. Pieza quirúrgica, masa de consistencia cauchosa de $21 \mathrm{~cm} \times 6 \mathrm{~cm}$.

Actualmente la paciente presenta evolución favorable, se mantiene en controles periódicos por nuestro servicio.

\section{DISCUSIÓN}

La LIV es un tipo raro y especial de tumor de músculo liso que afecta principalmente a mujeres premenopáusicas con antecedentes de miomas uterinos (4). El 90\% de los casos tienen síntomas relacionados con una masa pélvica, hipermenorrea, dolor pélvico $(2,3)$. Las 4 formas más frecuentes son: LIV que alcanza la vena cava inferior y puede extenderse a la aurícula derecha (leiomiomatosis intracardíaca) 
y arterias pulmonares, leiomioma metastásico benigno, leiomiomatosis peritoneal diseminada y leiomiomatosis hereditaria $(3,5)$.

A menudo no se reconocen en etapas tempranas, lo que lleva a un tratamiento inadecuado o tardío $(2,3)$. Cuando se produce una invasión extrapélvica, se se presentan con síntomas congestivos del lado derecho, entre los que se han descrito dolor torácico, disnea, edema de las extremidades inferiores, síncope y muerte súbita. La LIV tiene 4 etapas: estadio I, aquel que invade la vena uterina y se limita a la pelvis; se considera estadio II aquellos que se extienden a la cavidad abdominal, pero sin compromiso de la vena renal; estadio III si invaden la vena renal y la vena cava inferior extendiéndose hasta la aurícula derecha, pero sin llegar a las arterias pulmonares; y estadio IV, los tumores que llegan a las arterias pulmonares y/o hacen metástasis a los pulmones (4). El diagnóstico se suele realizar en estadios avanzados, cuando los síntomas son más evidentes y sugieren la necesidad de técnicas de imagen (2). Los métodos usados son la ecocardiografía, angiotomografía, resonancia magnética con contraste intravenoso; en el presente caso el diagnóstico se realizó mediante ecocardiografía, la cual detalló las características del tumor intracardiaco, así también, la angiotomografía reportó la invasión de vena cava y venas suprahepáticas (Figuras 1 y 2). El tratamiento, una vez establecido el diagnóstico, consiste en realizar la resección quirúrgica del tumor mediante un abordaje toracoabdominal con circulación extracorpórea e hipotermia, con el objetivo de realizar la resección tumoral completa. El procedimiento también se puede llevar a cabo en tiempos separados, con un intervalo no mayor de 6 semanas. Para los pacientes que no son candidatos para la resección quirúrgica se debe realizar supresión hormonal para evitar el crecimiento de los tumores.

Como conclusión, para la LIV que involucra al corazón, el diagnóstico preoperatorio oportuno y preciso, la preparación preoperatoria adecuada, y la selección razonable de métodos quirúrgicos son fundamentales para la resección tumoral completa. En este caso se trató de una LIV estadio III, que fue tratada con resección tumoral completa en el mismo tiempo quirúrgico con un excelente resultado.

\section{AGRADECIMIENTOS}

Un agradecimiento especial a todos los que forman el equipo de Cirugía Cardiovascular de la Clínica Guayaquil, encabezado por los Drs. Roberto y Mónica Gilbert, de igual manera al Departamento de Cirugía Oncológica encabezado por el Dr. Enrique Ortiz.

\section{REFERENCIAS BIBLIOGRÁFICAS}

1. Price JD, Anagnostopoulos C, Benvenisty A, Kothuru RK, Balaram SK. Intracardiac Extension of Intravenous Leiomyomatosis. Ann Thorac Surg. 2017;103(2):e145-7. DOI: 10.1016/j. athoracsur.2016.07.037

2. Valdés Devesa $V$, Conley CR, Stone WM, Collins JM, Magrina JF. Update on intravenous leiomyomatosis: Report of five patients and literature review. Eur J Obstet Gynecol Reprod Biol. 2013;171(2):209-13. DOI: 10.1016/j. ejogrb.2013.09.031

3. Declas E, Lucot JP. Extra uterine leiomyomatosis: Review of the literature. Gynecol Obstet Fertil Senol. 2019;47(7-8):582-90. DOI: 10.1016/j. gofs.2019.06.010

4. Yu X, Fu J, Cao T, Huang L, Qie M, Ouyang Y. Clinicopathologic features and clinical outcomes of intravenous leiomyomatosis of the uterus: A case series. Medicine (Baltimore). 2021;100(1):e24228. DOI: 10.1097/MD.0000000000024228

5. Luo G, Pan $\mathrm{H}$, Bi J, Luo Y, Zhu J, Feng Z, et al. Surgical treatment of intravenous leiomyomatosis involving the right heart: a case series. J Int Med Res. 2019;47(7):3465-74. DOI: $10.1177 / 0300060519858021$

\section{ACERCA DE LOS AUTORES}

1. Juan Gabriel Sarmiento Ortiz. Servicio de Cirugía General, Clínica Guayaquil, Guayaquil-Ecuador

ORCID: 0000-0002-2619-3651

2. Marcos Leonardo Matute Rivera. Servicio de Cirugía General, Clínica Guayaquil, Guayaquil-Ecuador

ORCID: 0000-0002-6867-0700

3. Luciana María Boloña Gilbert. Servicio de Cirugía General, Clínica Guayaquil, Guayaquil-Ecuador

ORCID: 0000-0002-0319-9882

4. Lorena Jaqueline Aragón Quijano. Servicio de Medicina Interna, Clínica Guayaquil, Guayaquil - Ecuador

\section{ORCID: 0000-0003-2403-0864}

5. Diana María Núñez Parra. Servicio de Cirugía General, Clínica Guayaquil, Guayaquil-Ecuador

ORCID: 0000-0002-2978-9311 